\title{
Alteração topográfica da curvatura corneana após aplicação de radiofreqüência em modelo animal
}

\author{
Corneal topographic alteration after radiofrequency application to an animal model
}

\author{
Leonardo Verri Paulino ${ }^{1}$ \\ Eduardo Paulino ${ }^{2}$ \\ Rodrigo Andrade de Barros 3 \\ Álvaro Gonçalves de Salles ${ }^{4}$ \\ José Ricardo Carvalho Lima Rehder ${ }^{5}$
}

\begin{tabular}{|c|}
\hline RESUMO \\
\hline $\begin{array}{l}\text { Objetivos: Verificar as alterações topográficas em córneas de olhos suínos } \\
\text { enucleados após aplicações de radiofreqüência. Métodos: Foi utilizado um } \\
\text { protótipo produtor de radiofreqüência, com } 10 \text { diferentes possibilidades de } \\
\text { potência. Para cada potência foram utilizados } 10 \text { olhos de porco enucleados } \\
\text { que foram topografados pré e pós-aplicação de radiofrequiência seguindo } \\
\text { nomograma com } 8 \text { pontos na primeira fase, } 16 \text { pontos na segunda fase, e } 24 \\
\text { pontos na terceira fase, somando } 3 \text { fases para cada olho, num total de } 300 \\
\text { aplicações e } 400 \text { topografias. Resultados: Ocorreram variações da curvatura } \\
\text { corneana após as aplicações de radiofreqüência para todas as potências } \\
\text { utilizadas, demonstradas pela variação das médias de KM (dioptria mínima) } \\
\text { iniciale KM final }(35,78 \text { e } 37,37 \text {, respectivamente) com uma variaçãode } 1,59 \\
\text { dioptrias (p< } 0,001) \text {. A potência que apresentou maior regularidade foi de } \\
0,625 \mathrm{~W} / \mathrm{s}\left(65^{\circ} \mathrm{C}\right) \text {. Conclusão: Os valores topográficos obtidos demonstram } \\
\text { que na média houve aumento da curvatura corneana central após aplicação } \\
\text { de radiofreqüência em olhos de porco enucleados, e que quanto maior a } \\
\text { energia utilizada e maior número de aplicações, maior foi o encurvamento } \\
\text { corneano, porém não respeitando um padrão homogêneo. }\end{array}$ \\
\hline
\end{tabular}

Descritores: Topografia da córnea; Erros de refração/cirurgia; Enucleação ocular; Ondas de rádio; Suínos; Modelos animais de doenças

\section{INTRODUCÃO}

Com os conhecimentos compartilhados hoje pela comunidade oftalmológica, muito tem sido feito para a resolução dos problemas ópticos mais comuns, como a miopia, a hipermetropia e o astigmatismo ${ }^{(1)}$.

Como o comprimento do globo ocular e o índice refrativo das estruturas oculares são unidades de difícil manipulação, a forma mais utilizada atualmente para a correção de ametropias é a modificação da curvatura corneana. No caso de um paciente com olho hipermétrope, a intenção é a de tornar a córnea mais curva centralmente ${ }^{(1-2)}$. As primeiras tentativas experimentais de modificação da estrutura corneana foram realizadas a mais de 100 anos, quando Lendert Jan Lans, publicou em $1898^{*}$ seu trabalho e os conceitos básicos deste trabalho permitiram o desenvolvimento, em meados da década de 60 , da técnica de ceratotomia radial ${ }^{(3)}$.

A termoceratoplastia também teve sua origem em experimentos de Lans, que em $1890^{* *}$ demonstrou que a aplicação de queimaduras térmicas super-

\footnotetext{
* Lans JL (1898). Experimental studies of astigmatic treatment with non perfurating corneal incisions. apud ${ }^{(3)}$ $* *$ Lans JL (1890) apud $\mathrm{d}^{(4-5)}$
} 
ficiais, em córneas de coelho, levava a alterações de sua curvatura $^{(4-5)}$. Com esta informação, 80 anos depois, estabeleceuse que a temperatura ideal para aplicação corneana estava em torno de $65^{\circ} \mathrm{C}$. Esta temperatura levava a um encolhimento das fibras de colágeno de $30 \%$ a $50 \%{ }^{(5-6)}$.

Em 1979, na tentativa de minimizar os efeitos colaterais da aplicação térmica na superfície corneana, criou-se um aparelho denominado "The Los Alamos Termoceratoplasty Probe", que utilizava pela primeira vez a radiofreqüência, aquecendo o colágeno no centro de córneas com ceratocone com intuito de diminuir sua curvatura ${ }^{(7-8)}$.

Na década de 80, desenvolveu-se em Moscou um procedimento que era um híbrido das técnicas de queimadura térmica corneana com o "Los Alamos Probe" de Rowsey, denominada termoceratoplastia radial. A técnica consistia de múltiplas aplicações de uma agulha retrátil profundamente no estroma corneano, que por 40 milisegundos liberava $600^{\circ} \mathrm{C}$ de calor; criando assim um encolhimento das fibras de colágeno que formava um cinturão circunferencial periférico, levando a um encurvamento central ${ }^{(9)}$

As técnicas de termoceratoplastia apresentam, em relação a ceratectomia fotorrefrativa, a vantagem da correção da hipermetropia sem manipular a zona óptica central. A desvantagem está relacionada à regressão progressiva do efeito da correção cirúrgica demonstrada com o passar dos anos ${ }^{(7-9)}$.

Estatisticamente, segundo o "The Baltimore Eye Study" realizado por pesquisadores da John Hopkins University, a hipermetropia afeta aproximadamente 60 milhões de pacientes acima de 40 anos nos EUA (o dobro em relação à miopia na mesma faixa etária) ${ }^{(10)}$.

Atualmente existem duas técnicas de termoceratoplastia liberadas para uso pelo FDA, o LTK (laser termal keratoplasty) com holmium:Yag laser e o CK (conductive keratoplasty) que utiliza radiofreqüência.

O objetivo deste trabalho é verificar as alterações topográficas em córneas de olhos enucleados de suínos após aplicações de radiofreqüência.

\section{MÉTODOS}

Este trabalho foi desenvolvido no Laboratório de Cirurgia Experimental do Departamento de Oftalmologia da Faculdade de Medicina da Faculdade de Medicina do ABC, entre o período de 15 de maio de 2002 a 15 de outubro de 2002, com o objetivo de verificar a previsibilidade das alterações topográficas após a aplicação de radiofreqüência em modelo animal, utilizando tecnologia nacional.

O objeto biológico utilizado no experimento foi o olho de porco enucleado. Para o emprego da radiofreqüência a empresa Loktal Medical Eletronics (São Paulo, SP) desenvolveu um aparelho experimental com as seguintes características:

- Freqüência de trabalho: $333 \mathrm{Khz}$

- Pólo ativo em uma caneta porta eletrodos

- Pólo passivo no blefarostato ou na placa de sucção

- Eletrodo ativo com filamento de aço inox nas dimensões de: comprimento $420 \mu$ - diâmetro $90 \mu$

\begin{tabular}{|cccc|}
\hline Posição da chave & Watts/segundo & Watts/60\% & Temperatura $\left({ }^{\circ} \mathbf{C}\right)$ \\
0 & 0,152 & 0,091 & 50 \\
1 & 0,220 & 0,132 & 52 \\
2 & 0,331 & 0,199 & 54 \\
3 & 0,415 & 0,249 & 56 \\
4 & 0,491 & 0,295 & 58 \\
5 & 0,567 & 0,340 & 61 \\
6 & 0,625 & 0,375 & 65 \\
7 & 0,726 & 0,436 & 66 \\
8 & 0,787 & 0,472 & 67 \\
9 & 0,874 & 0,524 & 70 \\
\hline
\end{tabular}

O topógrafo utilizado para a verificação das alterações de superfície corneana foi o modelo TMS-2 V2.2.3 da marca Tomey.

Para permitir uma reprodutibilidade da aplicação da radiofrequiência nos olhos de porco e sua avaliação pelo topógrafo foi desenvolvido um aparato de apresentação e transporte destes olhos. Este aparato constitui-se de uma caixa de isopor de $13,5 \mathrm{~cm}$ de altura por $15 \mathrm{~cm}$ de base e $7 \mathrm{~cm}$ de largura. Nesta caixa foi acoplada perpendicularmente, a $9 \mathrm{~cm}$ de altura e $4 \mathrm{~cm}$ no sentido horizontal, uma tampa plástica de bisnaga de centro cirúrgico ligada a uma seringa de $10 \mathrm{ml}$ através de um tubo de borracha flexível. Nesta mesma tampa plástica foi acondicionada uma placa de sucção do aparelho de radiofreqüência, que está conectada ao fio condutor passivo e uma cânula de borracha ligada a uma seringa de $20 \mathrm{ml}$.

As duas seringas acopladas servem como mecanismo de fixação do olho de porco ao sistema possibilitando uma manipulação e transporte mais seguro.

A metodologia constitui-se em colocar o olho de porco enucleado no aparato de transporte com seu maior diâmetro corneano na horizontal (maior diâmetro representa a posição nasal/ temporal pela anatomia corneana normal ${ }^{(1)}$ ); injetar $0,5 \mathrm{ml}$ de soro fisiológico $0,9 \%$ (SF) em câmara vítrea através de punção escleral com agulha de insulina, realizar uma desepitelização mecânica e, depois de umedecida a córnea por colírio lubrificante, topografar o olho. $\mathrm{O}$ aparato por suas características encaixa-se perfeitamente no topógrafo utilizado. (Figura 1a e 1b).

Obtida a topografia inicial, olho é submetido a uma secagem com hastes flexíveis de algodão.

A marcação dos pontos de aplicação de radiofreqüência foi realizada no centro óptico da córnea, com 7,0 mm de diâmetro e 8 segmentos de raio. O marcador utilizado foi o de cirurgias refrativas incisionais de Fyodorov e o centro óptico foi determinado através de reflexo luminoso.

Para cada posição de chave (nível de potência) do aparelho foram avaliados dez olhos de porco obedecendo ao seguinte nomograma:

- Fase 1 - oito aplicações de 0,60 segundos cada, realizadas exatamente a 7,0 $\mathrm{mm}$ de diâmetro nos oito pontos de cruzamento de raio.

- Fase 2 - oito aplicações de 0,60 segundos cada, no 

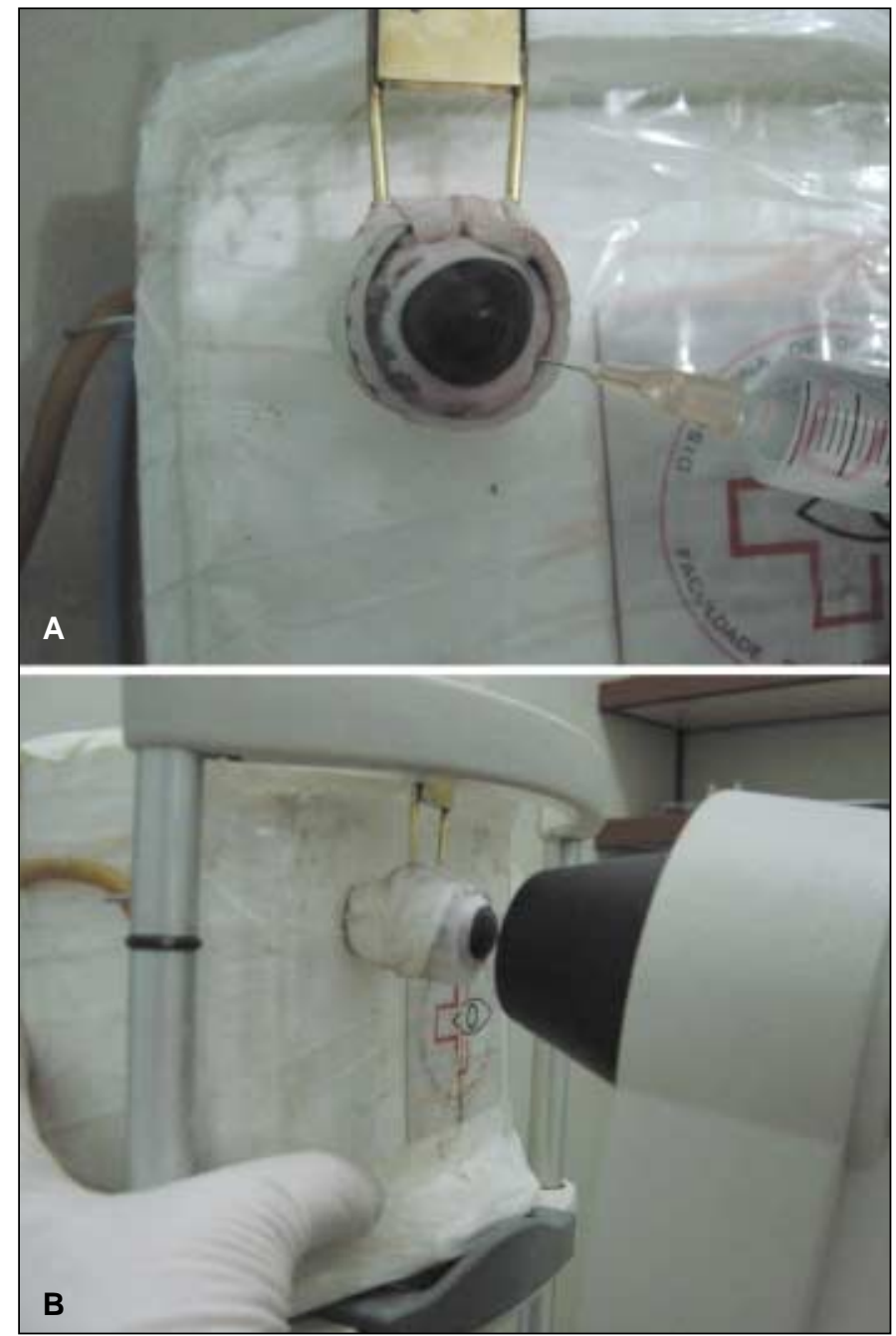

Figura 1 - A: Injeção de soro; B: Topografia em execução

diâmetro imediatamente externo dos mesmos pontos de raio, somando dezesseis aplicações.

- Fase 3 - oito aplicações de 0,60 segundos cada, no diâmetro imediatamente interno destes pontos de raio, totalizando vinte e quatro aplicações. (Figura 2 e 3 ).

Cada um dos 10 olhos passou pelas 3 fases de aplicação, sendo topografado antes da primeira fase, e entre cada fase. Portanto, para cada nível de potência foi realizado um total de 30 aplicações e 40 topografias (3 aplicações e 4 topografias para cada um dos 10 olhos), somando um total de 300 aplicações e 400 topografias ao final do trabalho.

A aplicação de SF antes do início do procedimento é necessária para aumentar a tonicidade do globo ocular que, pelo fato de ter sido enucleado 24 horas antes do procedimento, apresenta certa hipotonia. Isto possibilita a introdução da ponta de aplicação de radiofrequiência sem causar uma deformidade excessiva da córnea. O volume ideal desta infusão foi determinado por tonometria bidigital após testes de aplicação de variações volumétricas entre zero e $2 \mathrm{ml}$, com intervalos de $0,5 \mathrm{ml}$, realizados em 10 olhos não considerados no experimento.

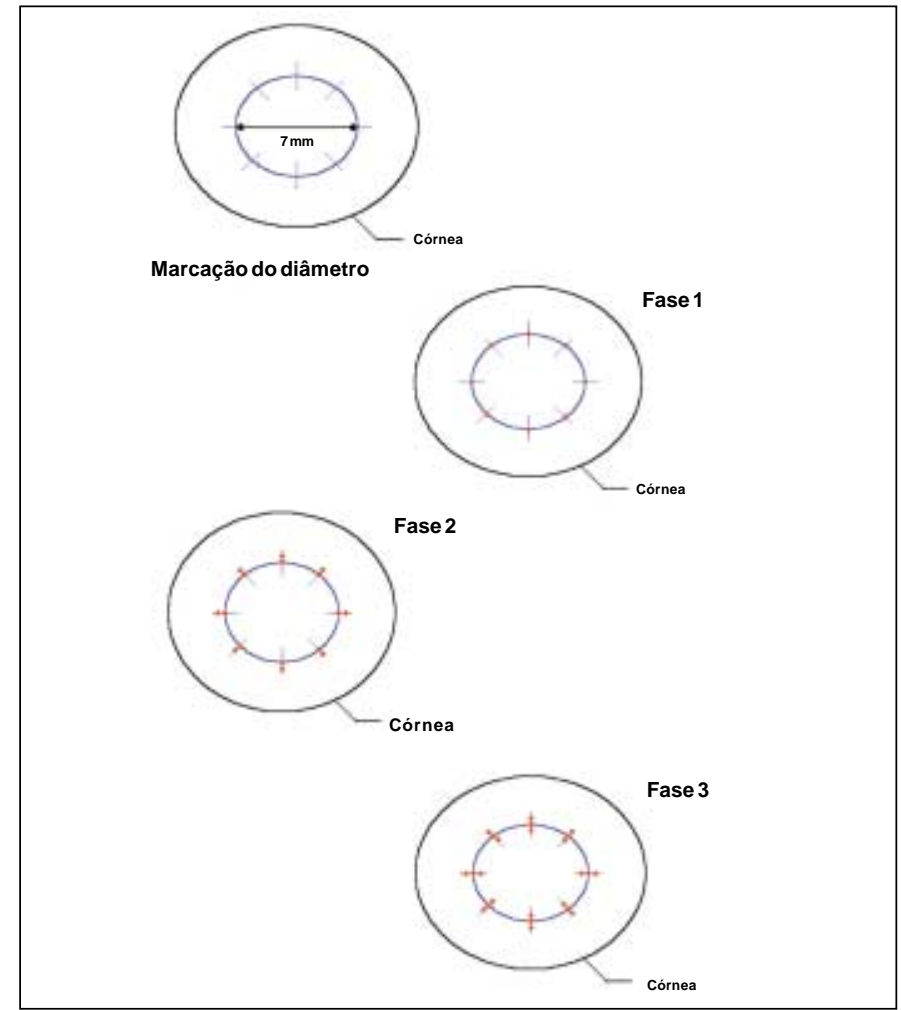

Figura 2 - Esquema de marcação e aplicações de radiofreqüência

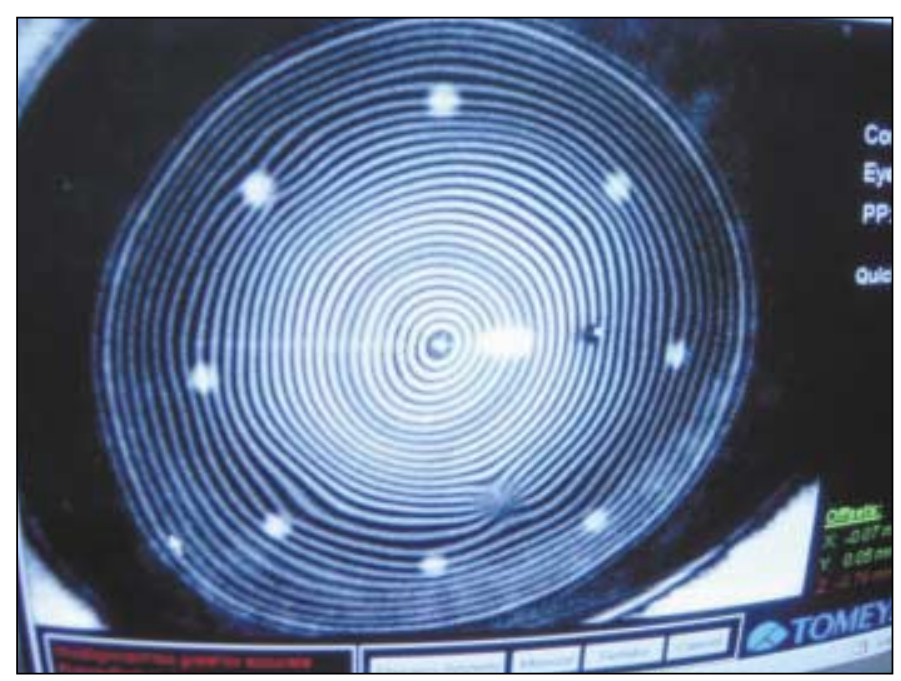

Figura 3 - Foto da topografia após Fase 1

A lubrificação ocular com o colírio regulariza a superfície corneana permitindo a realização da topografia com mais precisão, e a secagem é importante para que não ocorram perdas do poder energético da radiofrequiência por contato com o líquido durante a aplicação.

Entre cada uma das fases o olho foi umedecido, sendo submetido à nova secagem antes da próxima aplicação.

Foram montadas as planilhas de cada potência sendo inseridos os valores iniciais de K1 (dioptria mais curva), K2 (diop- 
tria mais plana) e KM (dioptria mínima) para cada um dos dez olhos avaliados, e subseqüentemente, os valores obtidos após aplicação energética em 8 pontos, 16 pontos e 24 pontos, progressivamente. Abaixo destes valores foram demonstrados a média aritmética e o desvio padrão de cada coluna, além da variação encontrada após cada aplicação. Após as três fases cada olho foi desprezado.

A análise dos resultados foi realizada através dos programas Statistica 5.0 e SPSS 10.0 for Windows. As comparações entre os grupos foram realizadas usando o teste $t$ de Student pareado devido as variáveis numéricas. Valores de p inferiores a 0,05 foram considerados significantes.

\section{RESULTADOS}

Os resultados topográficos obtidos pelos 10 olhos avaliados em cada potência testada estão demonstrados pela média aritmética e o desvio padrão de K1, K2 e KM, no início e após cada fase de aplicação (Tabela 1).

A média geral de KM inicial e KM após a terceira aplicação foi de 35,78 e 37,37, respectivamente, demonstrando uma variação positiva de curvatura de 1,59 dioptrias $(\mathrm{p}<0,001)$.

Nas posições $0,1,2$ e 4 da chave, ocorreram diminuição da curvatura central corneana em pelo menos uma das fases de aplicação. Este aplanamento verificado ocorreu de forma irregular e sem significância estatística.

Nas outras potências, em todas as aplicações, a curvatura corneana aumentou, porém as variações não se apresentaram de maneira uniforme.
A potência referente à posição " 6 " $\left(0,625 \mathrm{~W} / \mathrm{s}\right.$ ou $\left.65^{\circ} \mathrm{C}\right)$ apresentou uma variação entre KM inicial e final de 1,15, mantendo a maior regularidade e significância estatística entre as medidas aferidas. Isto se demonstra pela correlação da análise pareada entre as variáveis após cada a aplicação (Tabela 2).

Nesta mesma potência, quando comparado a média de K1 inicial e K1 após a terceira aplicação a variação foi de 2,10 ( $\mathrm{p}=0,007)$, de $\mathrm{K} 2$ inicial e K2 após a terceira aplicação variou $1,70(\mathrm{p}=0,009)$, e entre KM inicial e KM após a terceira aplicação foi de $3,10(\mathrm{p}=0,049)$.

A posição $8\left(0,787 \mathrm{~W} / \mathrm{s}\right.$ ou $\left.67^{\circ} \mathrm{C}\right)$, apesar de obter resultados de menor regularidade em relação à posição 6 , apresentou significância estatística entre a diferença da média de K1, K2 e KM inicial com K1, K2 e KM após a terceira aplicação (respectivamente de 4,30 dioptrias ( $p=0,001), 5,00(p=0,001)$ e 4,60 $(\mathrm{p}<0,001))$.

Para melhor visualização dos resultados foi desenvolvido um gráfico linear considerando-se os valores médios de KM do início ao fim das aplicações para todas as potências. (Gráfico 1)

\section{DISCUSSÃO}

A ceratoplastia condutiva $(\mathrm{CK})$ é um procedimento cirúrgico aprovado pelo FDA em 2002 para uso em hipermetropias entre $+0,75 \mathrm{a}+3,25$ dioptrias com até $-0,75$ de astigmatismo.

O conceito da CK é uma fusão dos conhecimentos adquiridos através da técnica de termoceratoplastia de Fyodorov com a tecnologia de radiofreqüência desenvolvida por JJ Rowsey. A primeira corrigia hipermetropias com queimaduras

\begin{tabular}{|c|c|c|c|c|c|c|c|c|c|c|c|c|}
\hline & \multicolumn{3}{|c|}{ Topografia Inicial } & \multicolumn{3}{|c|}{ Após 8 pontos } & \multicolumn{3}{|c|}{ Após 16 pontos } & \multicolumn{3}{|c|}{ Após 24 pontos } \\
\hline & K1 & K2 & KM & K1 & K2 & KM & K1 & K2 & KM & K1 & K2 & $\mathrm{KM}$ \\
\hline Posição 0 & $\begin{array}{l}40,46 \\
(3,27)\end{array}$ & $\begin{array}{l}35,95 \\
(3,21)\end{array}$ & $\begin{array}{c}34,94 \\
(2,69)\end{array}$ & $\begin{array}{l}42,10 \\
(3,25)\end{array}$ & $\begin{array}{l}37,96 \\
(3,60)\end{array}$ & $\begin{array}{l}36,55 \\
(3,21)\end{array}$ & $\begin{array}{l}41,35 \\
(3,11)\end{array}$ & $\begin{array}{l}37,18 \\
(2,54)\end{array}$ & $\begin{array}{l}35,74 \\
(2,47)\end{array}$ & $\begin{array}{c}42,66 \\
(4,03)\end{array}$ & $\begin{array}{l}37,49 \\
(3,99)\end{array}$ & $\begin{array}{c}35,76 \\
(3,52)\end{array}$ \\
\hline Posição 1 & $\begin{array}{l}41,19 \\
(1,81)\end{array}$ & $\begin{array}{l}37,17 \\
(2,76)\end{array}$ & $\begin{array}{l}35,98 \\
(2,63)\end{array}$ & $\begin{array}{l}40,12 \\
(1,60)\end{array}$ & $\begin{array}{l}37,12 \\
(2,52)\end{array}$ & $\begin{array}{l}35,57 \\
(2,88)\end{array}$ & $\begin{array}{l}41,49 \\
(2,16)\end{array}$ & $\begin{array}{l}37,12 \\
(2,06)\end{array}$ & $\begin{array}{l}36,01 \\
(1,88)\end{array}$ & $\begin{array}{l}41,87 \\
(3,55)\end{array}$ & $\begin{array}{l}36,96 \\
(3,14)\end{array}$ & $\begin{array}{l}35,47 \\
(3,34)\end{array}$ \\
\hline Posição 2 & $\begin{array}{l}40,88 \\
(1,20)\end{array}$ & $\begin{array}{l}37,94 \\
(2,04)\end{array}$ & $\begin{array}{l}37,17 \\
(2,10)\end{array}$ & $\begin{array}{l}42,89 \\
(1,71)\end{array}$ & $\begin{array}{l}37,99 \\
(2,72)\end{array}$ & $\begin{array}{l}37,11 \\
(1,94)\end{array}$ & $\begin{array}{l}45,15 \\
(2,59)\end{array}$ & $\begin{array}{l}39,80 \\
(2,46)\end{array}$ & $\begin{array}{c}38,82 \\
(2,18)\end{array}$ & $\begin{array}{l}44,30 \\
(1,85)\end{array}$ & $\begin{array}{l}39,34 \\
(3,07)\end{array}$ & $\begin{array}{c}36,60 \\
(3,38)\end{array}$ \\
\hline Posição 3 & $\begin{array}{l}38,44 \\
(3,23)\end{array}$ & $\begin{array}{l}34,47 \\
(3,33)\end{array}$ & $\begin{array}{l}33,12 \\
(3,28)\end{array}$ & $\begin{array}{l}39,44 \\
(2,24)\end{array}$ & $\begin{array}{l}34,75 \\
(3,94)\end{array}$ & $\begin{array}{l}33,59 \\
(3,34)\end{array}$ & $\begin{array}{l}39,63 \\
(1,49)\end{array}$ & $\begin{array}{l}35,11 \\
(2,23)\end{array}$ & $\begin{array}{l}34,12 \\
(2,39)\end{array}$ & $\begin{array}{l}41,24 \\
(1,89)\end{array}$ & $\begin{array}{l}37,42 \\
(3,07)\end{array}$ & $\begin{array}{c}36,06 \\
(2,84)\end{array}$ \\
\hline Posição 4 & $\begin{array}{l}41,28 \\
(1,71)\end{array}$ & $\begin{array}{c}38,09 \\
(2,20)\end{array}$ & $\begin{array}{l}36,80 \\
(1,90)\end{array}$ & $\begin{array}{l}42,00 \\
(1,06)\end{array}$ & $\begin{array}{l}38,17 \\
(2,10)\end{array}$ & $\begin{array}{l}36,97 \\
(2,41)\end{array}$ & $\begin{array}{c}43,29 \\
(1,90)\end{array}$ & $\begin{array}{c}39,52 \\
(1,92)\end{array}$ & $\begin{array}{l}37,80 \\
(1,59)\end{array}$ & $\begin{array}{c}43,60 \\
(1,28)\end{array}$ & $\begin{array}{l}39,36 \\
(3,29)\end{array}$ & $\begin{array}{c}37,35 \\
(2,72)\end{array}$ \\
\hline Posição 5 & $\begin{array}{c}40,36 \\
(2,62)\end{array}$ & $\begin{array}{l}35,80 \\
(2,06)\end{array}$ & $\begin{array}{l}35,68 \\
(1,96)\end{array}$ & $\begin{array}{l}40,28 \\
(1,97)\end{array}$ & $\begin{array}{l}37,02 \\
(1,81)\end{array}$ & $\begin{array}{l}36,11 \\
(1,59)\end{array}$ & $\begin{array}{l}40,65 \\
(2,34)\end{array}$ & $\begin{array}{l}37,29 \\
(2,45)\end{array}$ & $\begin{array}{l}36,41 \\
(2,34)\end{array}$ & $\begin{array}{c}42,23 \\
(3,36)\end{array}$ & $\begin{array}{l}38,66 \\
(3,17)\end{array}$ & $\begin{array}{c}36,81 \\
(3,16)\end{array}$ \\
\hline Posição 6 & $\begin{array}{l}40,46 \\
(1,21)\end{array}$ & $\begin{array}{c}36,53 \\
(2,73)\end{array}$ & $\begin{array}{l}36,06 \\
(2,80)\end{array}$ & $\begin{array}{l}40,90 \\
(1,50)\end{array}$ & $\begin{array}{l}37,03 \\
(2,99)\end{array}$ & $\begin{array}{l}36,30 \\
(3,12)\end{array}$ & $\begin{array}{l}41,52 \\
(2,21)\end{array}$ & $\begin{array}{l}37,92 \\
(1,39)\end{array}$ & $\begin{array}{l}36,68 \\
(2,18)\end{array}$ & $\begin{array}{c}42,43 \\
(2,44)\end{array}$ & $\begin{array}{l}38,29 \\
(2,75)\end{array}$ & $\begin{array}{l}37,21 \\
(2,83)\end{array}$ \\
\hline Posição 7 & $\begin{array}{l}41,00 \\
(1,78)\end{array}$ & $\begin{array}{l}38,21 \\
(1,81)\end{array}$ & $\begin{array}{l}37,36 \\
(1,61)\end{array}$ & $\begin{array}{l}41,64 \\
(1,69)\end{array}$ & $\begin{array}{l}38,95 \\
(1,84)\end{array}$ & $\begin{array}{l}37,53 \\
(2,41)\end{array}$ & $\begin{array}{l}41,08 \\
(1,67)\end{array}$ & $\begin{array}{l}38,49 \\
(2,32)\end{array}$ & $\begin{array}{l}37,22 \\
(2,94)\end{array}$ & $\begin{array}{l}46,02 \\
(2,41)\end{array}$ & $\begin{array}{l}40,84 \\
(2,77)\end{array}$ & $\begin{array}{c}38,89 \\
(2,75)\end{array}$ \\
\hline Posição 8 & $\begin{array}{c}41,28 \\
(3,60)\end{array}$ & $\begin{array}{c}35,85 \\
(2,45)\end{array}$ & $\begin{array}{l}34,47 \\
(2,06)\end{array}$ & $\begin{array}{l}42,81 \\
(2,01)\end{array}$ & $\begin{array}{l}38,88 \\
(2,21)\end{array}$ & $\begin{array}{c}37,38 \\
(2,10)\end{array}$ & $\begin{array}{l}43,58 \\
(5,25)\end{array}$ & $\begin{array}{c}38,89 \\
(3,18)\end{array}$ & $\begin{array}{l}37,44 \\
(3,25)\end{array}$ & $\begin{array}{c}45,81 \\
(2,90)\end{array}$ & $\begin{array}{l}40,86 \\
(3,21)\end{array}$ & $\begin{array}{c}38,98 \\
(2,38)\end{array}$ \\
\hline Posição 9 & $\begin{array}{l}40,69 \\
(2,16)\end{array}$ & $\begin{array}{l}36,50 \\
(3,83)\end{array}$ & $\begin{array}{c}36,26 \\
(4,09)\end{array}$ & $\begin{array}{l}43,66 \\
(3,79)\end{array}$ & $\begin{array}{l}37,09 \\
(1,70)\end{array}$ & $\begin{array}{l}35,97 \\
(2,70)\end{array}$ & $\begin{array}{c}42,78 \\
(2,70)\end{array}$ & $\begin{array}{l}38,71 \\
(4,33)\end{array}$ & $\begin{array}{l}37,98 \\
(4,15)\end{array}$ & $\begin{array}{c}49,19 \\
(5,01)\end{array}$ & $\begin{array}{l}42,66 \\
(5,38)\end{array}$ & $\begin{array}{l}40,57 \\
(4,34)\end{array}$ \\
\hline
\end{tabular}




\begin{tabular}{|c|c|c|}
\hline & Correlação & $\mathbf{p}$ \\
\hline $\mathrm{K} 1$ ini e $\mathrm{K} 1$ pri & 0,508 & 0,134 \\
\hline K1 pri e K1 seg & 0,640 & 0,46 \\
\hline $\mathrm{K} 1$ seg e $\mathrm{K} 1$ ter & 0,654 & 0,40 \\
\hline K2 ini e K2 pri & 0,839 & 0,002 \\
\hline K2 pri e K2 seg & 0,660 & 0,038 \\
\hline K2 seg e K2 ter & 0,750 & 0,012 \\
\hline KM ini e KM pri & 0,786 & 0,007 \\
\hline KM pri e KM seg & 0,910 & $<0,001$ \\
\hline $\mathrm{KM}$ seg e $\mathrm{KM}$ ter & 0,745 & 0,013 \\
\hline
\end{tabular}

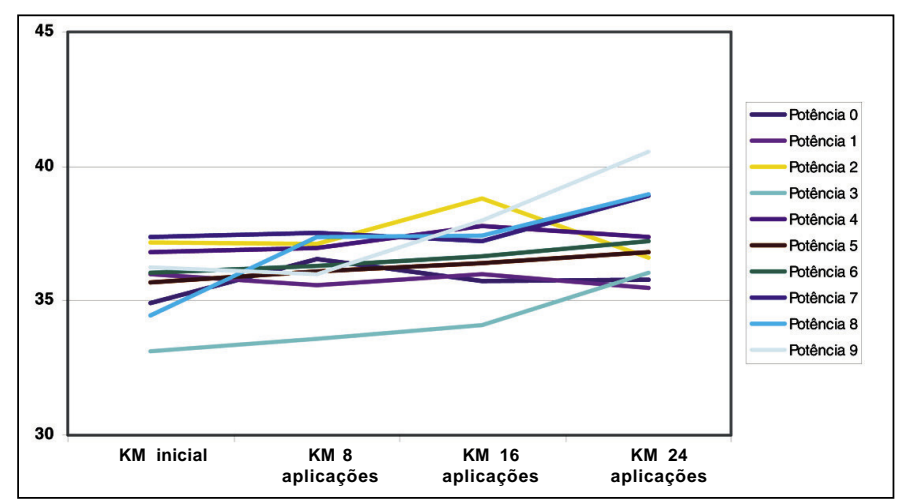

Gráfico 1 - Variação de curvatura em dioptrias para todas as potências

térmicas na periferia do estroma corneano, e a segunda utilizava um aplicador de radiofreqüência para aplanar o centro de ceratocones.

A utilização da radiofreqüência eleva a temperatura no estroma corneano de forma mais regular e controlada que a queimadura térmica, provocando um encolhimento das fibras de colágeno de forma mais duradoura, comprovado em pesquisas microscópicas ${ }^{(11)}$.Quando aplicada na periferia da córnea forma um cinturão circunferencial provocando um encurvamento central ${ }^{(9,12-13)}$.

Este estudo demonstra, de forma semelhante ao resultado obtido por experimento realizado em 2002 nos E.U.A. ${ }^{(14)}$, a possibilidade de aumento da curvatura corneana central após aplicação de radiofreqüência em sua periferia.

Observou-se que quanto maior a energia utilizada e maior número de aplicações, maior era o encurvamento corneano, porém não respeitando um padrão homogêneo. A potência que obteve maior regularidade no aumento de curvatura após as aplicações foi a de posição "6" $\left(0,625 \mathrm{~W} / \mathrm{s}\right.$ ou $\left.65^{\circ} \mathrm{C}\right)$, que confirma os resultados obtidos no trabalho de termoceratoplastia de $1970^{(5)}$.

Em algumas posições obtiveram-se variabilidades negativas após uma das aplicações de energia, além de diferentes respostas topográficas para aplicações de mesma potência em diferentes olhos de porco. Estas alterações justificam-se em função das condições peculiares de conservação de cada olho no momento do experimento, e da dificuldade técnica do exame topográfico dos mesmos.

\section{CONCLUSÃO}

Pelos dados obtidos no trabalho, seguindo a metodologia proposta, a média final das dioptrias demonstra um encurvamento corneano central em olhos suínos enucleados após a aplicação de radiofreqüência. Quanto maior a energia utilizada e maior número de aplicações, maior o encurvamento corneano, sem respeitar um padrão homogêneo.

\section{AGRADECIMENTOS}

À Lourdes da Conceição Martins, Auxiliar de Ensino do Departamento da Saúde da Coletividade da Faculdade de Medicina do $\mathrm{ABC}$, pelo auxílio na análise estatística.

\section{ABSTRACT}

Purpose: To verify the topographical alterations in enucleated pig eye cornea after radiofrequency application. Methods: A prototype radiofrequency modulator with 10 different energy possibilities was used. For each energy we used 10 enucleated pig eyes that were topographed before and after applyng radiofrequency, following a nomogram with 8 spots in step one, 16 spots in step 2, and 24 spots in step 3, in a total of 3 steps for each eye, 300 applications and 400 topographies. Results: Corneal curvature variation occurred after radiofrequency applications for all utilized selections, demonstrated by the mean variation of initial KM (minimum dioptry) and final KM (35.78 and 37.37 respectively) with 1.59 dioptries of variation $(\mathrm{p}<0.001)$. The energy that demonstrated more stability was $0.625 \mathrm{~W} / \mathrm{s}\left(65^{\circ} \mathrm{C}\right)$. Conclusion: The obtained topographic values demonstrated that the central corneal curvature increased after radiofrequency application, and the higher the energy and the more spots used, more curved became the cornea, despite homogeneity.

Keywords: Corneal topography; Refractive errors/surgery; Eye enucleation; Radio waves; Swine; Disease models, animal

\section{REFERÊNCIAS}

1. Smoler MK, Klyce SD. Cornea. In: Tasman W, Jaeger EA, editor. Duane's Ophthalmology [CD-ROM]. Philadelphia: JB Lippincott; 1995. p.8.

2. Sorsby A. Biology of the eye as an optical system. In: Tasman W, Jaeger EA, editor. Duane's Ophthalmology [CD-ROM]. Philadelphia: JB Lippincott; 1995. p.34.

3. Lane SS. Radial keratotomy surgical techniques. In: Krachmer JH, Mannis MJ, Holland EJ, Palay DA. Cornea text and color atlas. [CD-ROM]. St. Louis: Mosby; 1998. p.166.

4. Schallhorn SC, Mc Donnell PJ. Refractive surgery: past, present and future. In: Krachmer JH, Mannis MJ, Holland EJ, Palay DA. Cornea text and color atlas. [CD-ROM]. St. Louis: Mosby; 1998. p.161. 
5. Gasset AR, Kaufman HE. Thermokeratoplasty. Trans Am Acad Ophthalmol Otolaryngol. 1973;7(4):OP441-54.

6. Trokel SL, Srinivasan R, Braben B. Excimer laser surgery of the cornea. Am J Ophthalmol. 1983;96(6):710-5.

7. Rowsey JJ, Gaylor JR. Los Alamos keratoplasty techniques. Intraocul Lens Med. 1980;6(1):1-12

8. Rowsey JJ, Doss JD. Preliminary report of los Alamos keratoplasty techniques. Ophthalmology. 1981;88(8):755-60.

9. Caster AI. The Fyodorov technique of hyperopia correction by thermal coagulation: a preliminary report. J Refract Surg. 1988;4:105-8.

10. The Baltimore Eye Study. FDA approves surnrise technologies hyperion LTK system [text on the Internet]. [cited 2002 Jun 11]. Baltimore: John
Hopkins University; 1997. Available from:http://www.prk.com/SunriseTechPressRelease.html

11. Nishida T. Cornea. In: Krachmer JH, Mannis MJ, Holland EJ, Palay DA. Cornea text and colors atlas [CD-ROM]. Philadelphia: JB Lippincott; 1995. p.31.

12. Neumann AC, Fyodorov S, Sanders DR. Radial thermokeratoplasty for correction of hyperopia. Refract Corneal Surg. 1990;6(6):404-12.

13. Fyodorov S, Avashina A, Alexandrova OG. Analysis of surgical results of astigmatism correction by the technique of thermal coagulation and evaluation of accuracy of refractive effect prediction. Ophthalmol Surg. 1990;3(1):3-12.

14. Lussier J. Summary of safety and effectiveness data of ViewPoint ${ }^{\mathrm{tm}} \mathrm{CK}$ system: P010018. New York: FDA; 2002. 30p.

\title{
XXVI CONGRESSO DO HOSPITAL SÃO GERALDO
}

\section{2 a 04 de Novembro}

Ouro Minas Palace Hotel Belo Horizonte - MG

\author{
IN FORMAÇÕES: Consult - Comunicação e Eventos \\ Tel./ fax: (31) 3291-9899 \\ E-mail: consult@consultcom.com.br
}

